\title{
MICROPROPAGACIÓN DE DOS VARIEDADES ECUATORIANAS DE YUCA (Manihot esculenta CRANTZ)
}

\section{MICROPROPAGATION OF TWO ECUADORIAN VARIETIES OF YUCA (Manihot esculenta CRANTZ)}

\author{
Liliana Corozo ${ }^{1}$, Eduardo Héctor ${ }^{1,2}$, Fátima Macías ${ }^{1}$, Boris Vásquez $^{1}$, Bryan Pinargote $^{1}$, Gloria \\ Cobeña ${ }^{3}$, Alma Mendoza ${ }^{3}$, y Francisco Arteaga ${ }^{1}$
}

\author{
${ }^{1}$ Facultad de Ingeniería Agronómica, Universidad Técnica de Manabí, Portoviejo, Ecuador. \\ ${ }^{2}$ Instituto de Posgrado, Universidad Técnica de Manabí, Portoviejo, Ecuador. \\ ${ }^{3}$ Estación Experimental Portoviejo, Instituto Nacional de Investigaciones Agropecuarias (INIAP), \\ Portoviejo, Ecuador. \\ * Autor de correspondencia E-mail: lilita77corozo@gmail.com
}

\section{RESUMEN}

Se investigaron las condiciones esenciales para la micropropagación in vitro de las variedades ecuatorianas de yuca INIAP Portoviejo 650 e INIAP Portoviejo 651. Se plantó un banco de donantes con estacas de plantas vigorosas y sanas. En las etapas in vitro se empleó el medio de Murashige y Skoog (MS). En el establecimiento se adicionó Amoxicilina $500^{\circledast}$ y se evaluó la supervivencia de los explantes y la contaminación. En la multiplicación se utilizó $0,50 \mathrm{mg} \mathrm{L}^{-1}$ de 6-bencilaminopurina (6BAP) + 0,30-0,50 $\mathrm{mg} \mathrm{L}^{-1}$ de ácido giberélico $\left(\mathrm{GA}_{3}\right)$ y se evaluó la longitud de las plantas, el diámetro del tallo, el número de brotes y el número de hojas. Para el enraizamiento se empleó $1 \mathrm{mg} \mathrm{L}^{-1} \mathrm{de}$ ácido naftalenacético (ANA) + 0,01 $\mathrm{mg} \mathrm{L}^{-1}$ de ácido giberélico $\left(\mathrm{GA}_{3}\right)$, y se evaluó la longitud de las plantas, el diámetro del tallo, el número de raíces y la longitud de las raíces. En la aclimatación se evaluó la longitud de la planta, el diámetro del tallo, el número de brotes por planta y el número de hojas por planta. Se logró $\mathbf{1 0 0} \%$ de supervivencia en el establecimiento. En la multiplicación y el enraizamiento se observaron interacciones significativas variedad $x$ medio de cultivo, atribuibles al balance entre las fitohormonas presentes en los tejidos vegetales y los reguladores adicionados al medio. Se alcanzaron altos porcentajes de aclimatación (83-87\%). Se logró determinar las condiciones esenciales para micropropagar estas variedades de yuca, y se recomiendan estudios más profundos para estandarizar protocolos con vistas a su multiplicación masiva como vía de entrega de propágulos a los agricultores.

Palabras clave: cultivo in vitro, yuca, micropropagación

\section{ABSTRACT}

The essential conditions for the in vitro micropropagation of the Ecuadorian cassava varieties INIAP Portoviejo 650 and INIAP Portoviejo 651 were studied. A donor bank consisting of cuttings taken from vigorous, healthy plants was planted. In the in vitro stages, the medium of Murashige and Skoog (MS) was used. Amoxicillin $500^{\circledR}$ was added to the establishment and the survival of explants and contamination was evaluated. In multiplication, $0.50 \mathrm{mg} \mathrm{L}^{-1}$ of 6 -benzylaminopurine (6-BAP) + 0.30-0.50 $\mathrm{mg} \mathrm{L}^{-1}$ of gibberellic acid $\left(\mathrm{GA}_{3}\right)$ were used; plant height, stem diameter, and the number of shoots and leaves were evaluated. For rooting, $1 \mathrm{mg} \mathrm{L}^{-1}$ naphthalene acetic acid (ANA) $+0.01 \mathrm{mg} \mathrm{L}^{-1}$ gibberellic acid $\left(\mathrm{GA}_{3}\right)$ was used; the parameters evaluated were plant height, stem diameter, number of roots and root length. During acclimatization, plant height, stem diameter, 
and the number of shoots and leaves per plant were assessed. $100 \%$ survival was achieved in the establishment. In the multiplication and rooting, significant interactions were observed variety $x$ culture medium, attributable to the balance between the phytohormones present in the plant tissues and the regulators added to the medium. High percentages of acclimatization were reached (83-87\%). It was possible to determine the essential conditions for micropropagation of these cassava varieties, but more in-depth studies are recommended to standardize protocols with a view to their mass multiplication as a means of delivering propagules to farmers.

Key words: tissue culture, cassava, micropropagation

\section{INTRODUCCIÓN}

La yuca (Manihot esculenta Crantz) es un cultivo de origen neotropical, siendo las raíces tuberosas el órgano de interés comercial (Techeira et al., 2014). Se cultiva principalmente en el cinturón tropical y es considerado el mayor cultivo básico del mundo después del maíz (FAO, 2014). La producción mundial es de casi 280 millones de toneladas; aproximadamente el $61 \%$ de este total se cosecha en África, el $29 \%$ en Asia y el 9,8\% en América (FAOSTAT, 2018). En Ecuador se producen alrededor de $76892 \mathrm{t}$, de las cuales 12798 t corresponden a la provincia de Manabí (INEC, 2018).

El Instituto Nacional de Investigaciones Agropecuarias, Estación Experimental Portoviejo (INIAP-EEP) de Manabí, Ecuador, liberó en los años 1992 y 1998, las variedades INIAP Portoviejo 650 e INIAP Portoviejo 651, respectivamente, destinadas al consumo fresco, con contenidos de materia seca de alrededor del 35,5\% y baja tasa de conversión de almidón (7,5:1). Estas variedades tienen un rendimiento promedio de hasta el 25\% más que otras que se encuentran en la producción, y altos contenidos de amilasa y amilopectina, lo que permite obtener un almidón de alta calidad. Sin embargo, han sufrido los efectos de los factores bióticos y abióticos, afectando su altura, vigor, ramificación, producción de raíces y calidad de la semilla vegetativa, inconvenientes que están relacionados con el sistema de propagación tradicional (INIAP, 2008).

Tradicionalmente la yuca se propaga en forma vegetativa, pero este tipo de reproducción acarrea complicaciones como la transmisión de fitopatógenos (Mafla et al., 2010), baja productividad, alta heterocigosidad y baja fertilidad (Berhanu y Feyissa, 2013). La utilización de estaquillas (estacas) de mala calidad impide obtener plantaciones sanas, vigorosas y con buena producción; por esta razón se han buscado otras alternativas como el cultivo in vitro de tejidos para la propagación de la especie, lo que asegura sanidad vegetal, contribuye al intercambio internacional de germoplasma, y brinda material vegetal para la ingeniería genética (Roca, 1984; Souza et al. 2007; Akinbo et al., 2010; Liu et al.,
2011; Taylor et al., 2012; Mongomake et al., 2015).

Para lograr una germinación y un crecimiento satisfactorio en el cultivo in vitro de plantas, los medios de cultivo son determinantes; su composición debe ser muy precisa en cuanto a la adición de reguladores de crecimiento (Pereira et al., 2018), pues el balance entre estas sustancias determina el tipo de crecimiento y desarrollo de los tejidos y órganos vegetales. No existen protocolos para la multiplicación in vitro de las variedades de yuca INIAP Portoviejo 650 e INIAP Portoviejo 651, pudiendo ser una alternativa viable para su propagación acelerada. Por esa razón, el presente estudio tuvo como objetivo establecer las condiciones esenciales para la propagación in vitro de ambas variedades.

\section{MATERIALES Y MÉTODOS}

\section{Ubicación}

Las actividades de investigación fueron desarrolladas en el INIAP-EEP, y en el laboratorio de Cultivo de Tejidos de la Facultad de Ingeniería Agronómica de la Universidad Técnica de Manabí, Ecuador, en durante los años 2018 y 2019.

\section{Material vegetal}

Se seleccionaron estacas de las variedades de yuca INIAP Portoviejo 650 e INIAP Portoviejo 651, para lo cual se escogieron 10 plantas madre por cada variedad, del banco de germoplasma de yuca del INIAP-EEP, con madurez apropiada (8-12 meses), vigorosas, y visualmente libres de plagas y enfermedades. Para establecer un banco de donantes, del tercio medio de cada planta madre se cortaron tres estaquillas de aproximadamente $20 \mathrm{~cm}$ de longitud, que se desinfectaron sumergiéndolas por $5 \mathrm{~min}$ en una solución de Benomyl ${ }^{\circledast}\left(3 \mathrm{~g} \mathrm{~L}^{-1}\right)+$ Clorpirifos $^{\circledast}(2 \mathrm{ml}$ $\left.\mathrm{L}^{-1}\right)$. Las estaquillas se sembraron en un vivero, en fundas de polietileno ( $\left.5 \times 7^{\prime \prime}\right)$ perforadas, que contenían sustrato (suelo + arena de río 2:1) previamente esterilizado en autoclave por dos horas. Para el mantenimiento de las estacas se realizaron riegos frecuentes con solución nutritiva de Hoagland (Beltrano y Gimenez, 2015) para acelerar el desarrollo de las yemas. 


\section{Establecimiento in vitro}

Se utilizaron brotes tiernos de plantas de cuatro semanas de edad procedentes del vivero. Se tomaron porciones de tallo de $2-3 \mathrm{~cm}$ que incluían una yema, que se lavaron en el laboratorio con jabón líquido por 5 min y se enjuagaron con abundante agua corriente. Posteriormente, en la cámara de flujo laminar, las yemas se colocaron en etanol $70 \%$ por $30 \mathrm{~s}$, luego en hipoclorito de sodio al $2 \%$ por $15 \mathrm{~min}$, y finalmente se lavaron con agua destilada esterilizada hasta eliminar residuos de hipoclorito de sodio. Se retiraron hojas y estípulas, y se establecieron 50 ápices de cada variedad en tubos de ensayo con medio de cultivo (Murashige y Skoog, 1962) con Amoxicilina 500 $\left(5 \mathrm{mg} \mathrm{L}^{-1}\right)$. Los tubos se colocaron en la cámara de crecimiento por 30 días, con fotoperiodo de 16 horas luz y 8 de oscuridad, y temperatura de $24 \pm 2^{\circ} \mathrm{C}$. En esta fase se evaluó el porcentaje de supervivencia y el número de explantes contaminados por hongos y bacterias.

\section{Multiplicación in vitro}

Los brotes se transfirieron a tubos de ensayo con medio de cultivo de Murashige y Skoog (1962) suplementado o no con 6-bencilaminopurina (BAP) y ácido giberélico $\left(\mathrm{GA}_{3}\right)$ (Tabla 1). Los cultivos se mantuvieron en las mismas condiciones indicadas en la fase de establecimiento y se evaluó la longitud de las plantas $(\mathrm{cm})$, el diámetro del tallo $(\mathrm{cm})$, el número de brotes y de hojas en 5 subcultivos de 30 días cada uno. Se utilizó un Diseño Completamente al Azar (DCA), en arreglo factorial $2 \times 4$ (variedades $\mathrm{x}$ tratamientos) y 3 explantes por tratamiento.

\section{Enraizamiento in vitro}

Se seleccionaron explantes vigorosos procedentes de la última multiplicación y se transfirieron a tubos de ensayo con medio de cultivo de Murashige y Skoog (1962) suplementado o no con ácido naftalenacético (ANA) y ácido giberélico $\left(\mathrm{GA}_{3}\right)$ (Tabla 1$)$. Los cultivos se mantuvieron en iguales condiciones de temperatura y fotoperíodo que en las fases anteriores durante 30 días de cultivo. En esta fase se evaluó la longitud de las plantas $(\mathrm{cm})$, el diámetro del tallo $(\mathrm{cm})$, el número de raíces y la longitud de las raíces $(\mathrm{cm})$. Se utilizó un Diseño Completamente al Azar (DCA), en arreglo factorial $2 \times 4$ (variedades $\times$ tratamientos) y 15 explantes por tratamiento.

\section{Aclimatación}

Para adaptar las vitroplantas a condiciones de humedad ambiental, cinco días antes del trasplante se eliminó el film que cubría al tubo, luego se retiró la plántula del tubo y se lavaron las raíces con abundante agua, hasta eliminar por completo el medio de cultivo. A continuación, se sembraron 30 vitroplantas por cada variedad, en bandejas de plástico de 50 alveolos $(55 \mathrm{~cm}$ de largo, $29 \mathrm{~cm}$ de ancho y 7,5 cm de alto) que contenían turba (sustrato). Una vez sembradas las plantas se cubrieron con vasos de espuma flex durante dos semanas, con el objeto de formar una cámara húmeda y evitar deshidratación. Se aplicó semanalmente la solución nutritiva de Hoagland (macro y micronutrientes). Las plántulas permanecieron en casa de malla durante 60 días y se evaluó la longitud de la planta $(\mathrm{cm})$, el

Tabla 1. Tratamientos aplicados en la propagación in vitro de variedades de yuca.

Table 1. Treatments applied in the in vitro propagation of cassava varieties.

\section{Fase de multiplicación}

\begin{tabular}{|c|c|}
\hline Variedad INIAP Portoviejo 650 (V1) & Variedad INIAP Portoviejo 651 (V2) \\
\hline $\mathrm{T} 1\left(\mathrm{~V} 1 \mathrm{M} 1=0,50 \mathrm{mg} \mathrm{L}^{-1} \mathrm{BAP}+0,30 \mathrm{mg} \mathrm{L}^{-1} \mathrm{GA}_{3}\right)$ & $\mathrm{T} 5\left(\mathrm{~V} 2 \mathrm{M} 1=0,5 \mathrm{mg} \mathrm{L}^{-1} \mathrm{BAP}+0,30 \mathrm{mg} \mathrm{L}^{-1} \mathrm{GA}_{3}\right)$ \\
\hline $\mathrm{T} 2\left(\mathrm{~V} 1 \mathrm{M} 2=0,50 \mathrm{mg} \mathrm{L}^{-1} \mathrm{BAP}+0,40 \mathrm{mg} \mathrm{L}^{-1} \mathrm{GA}_{3}\right)$ & $\mathrm{T} 6\left(\mathrm{~V} 2 \mathrm{M} 2=0,5 \mathrm{mg} \mathrm{L}^{-1} \mathrm{BAP}+0,40 \mathrm{mg} \mathrm{L}^{-1} \mathrm{GA}_{3}\right)$ \\
\hline $\mathrm{T} 3\left(\mathrm{~V} 1 \mathrm{M} 3=0,50 \mathrm{mg} \mathrm{L}^{-1} \mathrm{BAP}+0,50 \mathrm{mg} \mathrm{L}^{-1} \mathrm{GA}_{3}\right)$ & $\mathrm{T} 7\left(\mathrm{~V} 2 \mathrm{M} 3=0,5 \mathrm{mg} \mathrm{L}^{-1} \mathrm{BAP}+0,50 \mathrm{mg} \mathrm{L}^{-1} \mathrm{GA}_{3}\right)$ \\
\hline $\begin{array}{l}\mathrm{T} 4(\mathrm{~V} 1 \mathrm{M} 0=\text { testigo } \sin \text { reguladores del } \\
\text { crecimiento) }\end{array}$ & $\begin{array}{l}\mathrm{T} 8(\mathrm{~V} 2 \mathrm{M} 0=\text { testigo sin reguladores del } \\
\text { crecimiento) }\end{array}$ \\
\hline
\end{tabular}

Fase de enraizamiento

\begin{tabular}{ll}
\hline Variedad INIAP Portoviejo $650(\mathrm{~V} 1)$ & Variedad INIAP Portoviejo 651 $(\mathrm{V} 2)$ \\
$\mathrm{T} 1\left(\mathrm{~V} 1 \mathrm{M} 1=1 \mathrm{mg} \mathrm{L}^{-1} \mathrm{ANA}+0,01 \mathrm{mg} \mathrm{L}^{-1} \mathrm{GA}_{3}\right)$ & $\mathrm{T} 3\left(\mathrm{~V} 2 \mathrm{M} 1=1 \mathrm{mg} \mathrm{L}^{-1} \mathrm{ANA}+0,01 \mathrm{mg} \mathrm{L}^{-1} \mathrm{GA}_{3}\right)$ \\
$\mathrm{T} 2(\mathrm{~V} 1 \mathrm{M} 0=$ testigo sin reguladores del & $\mathrm{T} 4(\mathrm{~V} 2 \mathrm{M} 0=$ testigo sin reguladores del \\
crecimiento $)$ & crecimiento $)$
\end{tabular}

T: Tratamiento; M: Medios de cultivo; V: Variedades de yuca

BAP: 6-Bencilaminopurina

$\mathrm{GA}_{3}$ : Ácido giberélico

ANA: Ácido naftalenacético 
diámetro del tallo $(\mathrm{cm})$, el número de brotes y número de hojas por planta.

\section{Análisis estadístico}

En la fase de establecimiento se registraron los porcentajes de ápices vivos y no contaminados (sanos). En las fases de multiplicación y enraizamiento in vitro fueron analizadas a través de un análisis de varianza (ANOVA) para dos factores (variedad $x$ medio de cultivo), aplicando la prueba de comparación múltiple de Tukey $(\mathrm{p}<0,05)$ para las medias de los tratamientos. En la fase de aclimatación, la altura de planta, el diámetro del tallo, el número de brotes y el número de hojas, se analizaron mediante una prueba de T-Student $(\alpha=0,05)$, mientras que los porcentajes de supervivencia se compararon a través de la prueba de Kaplan- Meier (estadístico de prueba Log Rank). Los análisis se realizaron con los paquetes estadísticos InfoStat e IMB SPSS Statistics 21.

\section{RESULTADOS Y DISCUSIÓN}

\section{Establecimiento in vitro}

Los explantes de las variedades INIAP Portoviejo 650 e INIAP Portoviejo 651 alcanzaron el máximo porcentaje de supervivencia $(100 \%)$ y no se observó contaminación microbiana en la siembra, lo cual podría deberse al proceso de desinfección empleado y a la adición al medio de cultivo del antibiótico Amoxicilina $500^{\circledast}$. Abreu et al. (2016) mencionaron que el uso de antibióticos como suplemento para los medios de cultivo es esencial en la eliminación de la contaminación microbiana, especialmente bacterias. Esto ha sido corroborado por otros autores (Reddy et al., 2010; Leelavathy y Sankar, 2016), quienes indicaron que añadir la cantidad apropiada de antibióticos al medio de cultivo puede evitar la contaminación en los explantes sembrados. Asimismo, fue posible regenerar plantas a partir de las dos variedades, lográndose que el $100 \%$ de los explantes se desarrollaran normalmente en cuanto a su tamaño, forma y coloración.

\section{Multiplicación in vitro}

En la longitud de las plantas, se pudo apreciar diferencias significativas para la interacción entre las variedades y los medios $(\mathrm{VxM})$. Los mayores valores para esta variable $(2,48 \mathrm{~cm}$ y $2,47 \mathrm{~cm})$ se obtuvieron en las combinaciones V2M1 y V1M3, respectivamente (Tabla 2 ).

La longitud de las plantas es una variable importante en la micropropagación, ya que plantas más altas tienen un mayor número de

Tabla 2. Efecto de los medios de cultivo sobre la longitud de la planta, el diámetro del tallo, el número de brotes y de hojas de dos variedades de yuca multiplicadas in vitro.

Table 2. Effect of culture medium on plant height, stem diameter, and number of shoots and leaves of two varieties of cassava multiplied in vitro.

\begin{tabular}{lcccc}
\hline Factores & $\begin{array}{c}\text { Longitud de la } \\
\text { planta }(\mathrm{cm})\end{array}$ & $\begin{array}{c}\text { Diámetro del } \\
\text { tallo }(\mathrm{cm})\end{array}$ & $\begin{array}{c}\text { Número de } \\
\text { brotes }\end{array}$ & $\begin{array}{c}\text { Número de } \\
\text { hojas }\end{array}$ \\
\hline Variedades & & & & \\
V1: INIAP Portoviejo 650 & $2,07 \pm 0,08$ & $0,23 \pm 0,01$ & $3,13 \pm 0,23$ & $3,50 \pm 0,29 \mathrm{~b}$ \\
V2: INIAP Portoviejo 651 & $1,88 \pm 0,09$ & $0,24 \pm 0,01$ & $3,35 \pm 0,22$ & $4,15 \pm 0,25 \mathrm{a}$ \\
\hline Medios & & & & \\
M1: BAP 0,5 mg L-1 $+\mathrm{GA}_{3} 0,30 \mathrm{mg} \mathrm{L}^{-1}$ & $2,22 \pm 0,11$ & $0,23 \pm 0,01$ & $3,60 \pm 0,39$ & $4,20 \pm 0,45$ \\
M2: BAP 0,5 $\mathrm{mg} \mathrm{L}^{-1}+\mathrm{GA}_{3} 0,40 \mathrm{mg} \mathrm{L}^{-1}$ & $1,96 \pm 0,11$ & $0,23 \pm 0,02$ & $3,20 \pm 0,30$ & $3,80 \pm 0,44$ \\
M3: BAP 0,5 $\mathrm{mg} \mathrm{L}^{-1}+\mathrm{GA}_{3} 0,50 \mathrm{mg} \mathrm{L}^{-1}$ & $1,93 \pm 0,16$ & $0,23 \pm 0,01$ & $3,05 \pm 0,30$ & $3,55 \pm 0,27$ \\
M4: Testigo sin reguladores del & & & \\
crecimiento & $1,80 \pm 0,07$ & $0,25 \pm 0,02$ & $3,10 \pm 0,27$ & $3,75 \pm 0,38$ \\
\hline Interacción & & & & \\
V1 M1 & $1,95 \pm 0,17 \mathrm{abc}$ & $0,20 \pm 0,02$ & $2,50 \pm 0,58 \mathrm{c}$ & $3,10 \pm 0,72 \mathrm{~b}$ \\
V1 M2 & $2,01 \pm 0,20 \mathrm{ab}$ & $0,23 \pm 0,03$ & $3,00 \pm 0,49 \mathrm{bc}$ & $3,50 \pm 0,75 \mathrm{ab}$ \\
V1 M3 & $2,47 \pm 0,13 \mathrm{a}$ & $0,24 \pm 0,02$ & $4,10 \pm 0,31 \mathrm{ab}$ & $4,20 \pm 0,36 \mathrm{ab}$ \\
V1 M4 & $1,83 \pm 0,07 \mathrm{bc}$ & $0,24 \pm 0,02$ & $2,90 \pm 0,23 \mathrm{bc}$ & $3,20 \pm 0,42 \mathrm{~b}$ \\
V2 M1 & $2,48 \pm 0,10 \mathrm{a}$ & $0,26 \pm 0,02$ & $4,70 \pm 0,21 \mathrm{a}$ & $5,30 \pm 0,26 \mathrm{ab}$ \\
V2 M2 & $1,90 \pm 0,08 \mathrm{bc}$ & $0,22 \pm 0,01$ & $3,40 \pm 0,34 \mathrm{abc}$ & $4,10 \pm 0,50 \mathrm{ab}$ \\
V2 M3 & $1,38 \pm 0,17 \mathrm{c}$ & $0,22 \pm 0,01$ & $2,00 \pm 0,21 \mathrm{c}$ & $2,90 \pm 0,28 \mathrm{~b}$ \\
V2 M4 & $1,76 \pm 0,12 \mathrm{bc}$ & $0,25 \pm 0,03$ & $3,30 \pm 0,50 \mathrm{abc}$ & $4,30 \pm 0,62 \mathrm{ab}$ \\
\hline
\end{tabular}


nudos, se facilita el corte y se obtienen más explantes para la propagación siguiente. Yandia et al. (2018) reportaron una altura promedio de 5,28 $\mathrm{cm}$ en el cultivar Yalipe de yuca cultivado en MS + ANA $\left(0,02 \mathrm{mg} \mathrm{L}^{-1}\right)+\operatorname{BAP}\left(0,05 \mathrm{mg} \mathrm{L}^{-1}\right)+\mathrm{GA} 3(0,02$ $\mathrm{mg} \mathrm{L}^{-1}$ ). Mehmood et al. (2016) observaron que las mayores longitudes en plantas de dos variedades de papa $(10,3 \mathrm{~cm}$ y $10 \mathrm{~cm})$ se obtuvieron al aplicar 0,25 y $0,5 \mathrm{mg} \mathrm{L}^{-1} \mathrm{de} \mathrm{GA}_{3}$ al medio de cultivo, pero concentraciones superiores produjeron plántulas débiles y tiernas.

En el diámetro del tallo no se obtuvieron diferencias entre las variedades, los medios de cultivo o sus interacciones. Los valores promedio de esta variable oscilaron entre 0,20 y $0,26 \mathrm{~cm}$; estos resultados coinciden con los reportados por Collado et al. (2004) en yuca, que obtuvieron un promedio de $0,20 \mathrm{~cm}$ de diámetro empleando 2 $\mathrm{mg} \mathrm{L}^{-1}$ de BAP $+0,2 \mathrm{mg} \mathrm{L}^{-1}$ de $\mathrm{GA}_{3}$.

En el número de brotes también se observó interacción $\mathrm{VxM}$, y cuatro de los tratamientos condujeron a los mayores valores para esta variable, a un mismo nivel de significación (V1M3, V2M1, V2M2, V2M4). Cabe resaltar, que las dos variedades respondieron de manera diferente según el medio de cultivo empleado; mientras para INIAP Portoviejo 650 los mayores valores se obtuvieron con la mayor concentración de $\mathrm{GA}_{3}\left(0,50 \mathrm{mg} \mathrm{L}^{-1}\right)$, en cuanto a la variedad INIAP Portoviejo 651 respondió de igual manera cuando no se añadió $\mathrm{GA}_{3}$ al medio (M4) o cuando se emplearon concentraciones menores (M1 y M2), en cambio la concentración mayor (M3) produce valores significativamente inferiores. Estos resultados son interesantes, pues en otras especies como la papa, el número de brotes obtenidos no depende de la concentración de $\mathrm{GA}_{3}$ en el medio de cultivo (Rabbani et al., 2001; Ali et al., 2018). En yuca, Moura y Ferreira (2004) mencionaron que en la propagación in vitro la respuesta de los esquejes de cuatro cultivares dependió del genotipo, pero no de la concentración de $\mathrm{GA}_{3}$.

En el número de hojas por planta, se hallaron diferencias significativas en las interacciones $\mathrm{VxM}$ y en las variedades. Al observar los valores estadísticos para este factor, se aprecia que INIAP Portoviejo 651 obtuvo valores significativamente superiores a los de INIAP Portoviejo 650, lo que debe tener su explicación en las diferencias genéticas entre los dos genotipos. Las hojas son el sitio principal de producción de sustancias elaboradas para la planta, y un sistema de hojas bien desarrollado será importante para la supervivencia de la planta durante la aclimatación (Ogero et al., 2012). Lolaei et al. (2013) mencionaron que el GA interviene en gran medida en las etapas de crecimiento y desarrollo de las plantas y aumenta el número de hojas cuando se aplica en bajas concentraciones. $\mathrm{Xu}$ et al. (2016) señalaron que el tratamiento con $\mathrm{GA}_{3}$ es determinante en la elongación de las hojas y su productividad.

\section{Enraizamiento in vitro}

También en esta fase se observaron diferencias significativas atribuibles a la interacción VxM (Tabla 3) en todas las variables. No se obtuvieron raíces en ninguna de las variedades cuando se utilizó medio de cultivo sin reguladores del crecimiento.

Tabla 3. Efecto de ANA y GA $\mathrm{G}_{3}$ en la longitud de la planta, diámetro del tallo, número de raíces y longitud de las raíces en el enraizamiento in vitro de explantes de yuca.

Table 3. Effect of ANA and $\mathrm{GA}_{3}$ on plant height, stem diameter, number of roots and root length in the in vitro rooting of cassava explants.

\begin{tabular}{|c|c|c|c|c|}
\hline Factores & $\begin{array}{l}\text { Longitud de } \\
\text { la planta }(\mathrm{cm})\end{array}$ & $\begin{array}{l}\text { Diámetro del } \\
\text { tallo }(\mathrm{cm})\end{array}$ & $\begin{array}{l}\text { Número de } \\
\text { raíces }\end{array}$ & $\begin{array}{l}\text { Longitud de } \\
\text { las raíces }(\mathrm{cm})\end{array}$ \\
\hline \multicolumn{5}{|l|}{ Variedades } \\
\hline V1: INIAP Portoviejo 650 & $1,99 \pm 0,15$ & $0,24 \pm 0,01 \mathrm{a}$ & $4,43 \pm 0,90$ & $0,40 \pm 0,09 \mathrm{~b}$ \\
\hline V2: INIAP Portoviejo 651 & $1,72 \pm 0,09$ & $0,22 \pm 0,01 b$ & $3,90 \pm 0,77$ & $0,70 \pm 0,16 \mathrm{a}$ \\
\hline \multicolumn{5}{|l|}{ Medios } \\
\hline M1: ANA $1 \mathrm{~m} \mathrm{gL}^{-1}+\mathrm{GA}_{3} 0,01 \mathrm{mg} \mathrm{L}^{-1}$ & $1,54 \pm 0,11 \mathrm{~b}$ & $0,20 \pm 0,00 \mathrm{~b}$ & $8,33 \pm 0,47$ a & $1,11 \pm 0,11 \mathrm{a}$ \\
\hline $\begin{array}{l}\text { M2: Testigo sin reguladores del } \\
\text { crecimiento }\end{array}$ & $2,18 \pm 0,12 \mathrm{a}$ & $0,26 \pm 0,01 \mathrm{a}$ & $0,00 \pm 0,00 \mathrm{~b}$ & $0,00 \pm 0,00 \mathrm{~b}$ \\
\hline \multicolumn{5}{|l|}{ Interacción } \\
\hline V1 M1 & $1,37 \pm 1,12 b$ & $0,20 \pm 0,00 \mathrm{~b}$ & $8,87 \pm 0,74$ a & $0,81 \pm 0,08 \mathrm{~b}$ \\
\hline V1 M2 & $2,61 \pm 0,18 \mathrm{a}$ & $0,29 \pm 0,02 \mathrm{a}$ & $0,00 \pm 0,00 \mathrm{~b}$ & $0,00 \pm 0,00 \mathrm{c}$ \\
\hline V2 M1 & $1,70 \pm 0,18 b$ & $0,21 \pm 0,01 b$ & $7,80 \pm 0,55 \mathrm{a}$ & $1,41 \pm 0,18 \mathrm{a}$ \\
\hline V2 M2 & $1,75 \pm 0,05 \mathrm{~b}$ & $0,23 \pm 0,01 b$ & $0,00 \pm 0,00 \mathrm{~b}$ & $0,00 \pm 0,00 \mathrm{c}$ \\
\hline
\end{tabular}


Ulloa et al. (2017) y Kidulile et al. (2018) obtuvieron los mayores valores en la longitud de las plantas de yuca sin reguladores del crecimiento. Ulloa et al. (2017) lograron hasta 6,3 raíces por planta en medio de cultivo sin reguladores del crecimiento, lo cual no concuerda con lo obtenido en la presente investigación; sin embargo, el efecto inductor de auxinas como el ANA en la producción de raíces ha sido ampliamente estudiado (Taiz y Zeiger 2006; Palla et al., 2013; Elmongy et al., 2018). Es posible también que la presencia de $\mathrm{GA}_{3}$ en el medio de cultivo, por la función que ejerce esta sustancia en la promoción del crecimiento del tallo, las hojas y raíces (Ortega et al., 2013), haya contribuido a los resultados obtenidos.

En general, en las variables evaluadas en las etapas de multiplicación y enraizamiento se observaron interacciones VxM. Como norma, en los laboratorios de cultivo in vitro no se determinan las concentraciones de fitohormonas presentes en los tejidos vegetales; sin embargo, estas sustancias interactúan con los reguladores del crecimiento que se añaden a los medios de cultivo, dando lugar a estos comportamientos, que resultan del balance entre los reguladores endógenos y exógenos. Este fenómeno ha sido demostrado en otras especies (Héctor et al., 2007; Basail, 2015) y también en yuca (Suárez y Hernández, 2015).

\section{Aclimatación}

La supervivencia en esta etapa fue de $87 \%$ para la variedad INIAP Portoviejo 650 y de $83 \%$ para la variedad INIAP Portoviejo 651 , sin diferencias estadísticas entre ellas. Este es un resultado muy favorable, pues las pérdidas en la etapa de aclimatación son críticas en el proceso de micropropagación (Segovia et al., 2002). La yuca es una de las especies que sufren pérdidas importantes en esta fase (Laynez y Sánchez, 2006; Marín et al., 2009), pues las plantas deben ajustar su fisiología estomática a las condiciones de evapotranspiración imperantes y activar la toma de agua y nutrientes a través del sistema radical (Bonilla et al., 2015). En las restantes variables evaluadas no se observaron diferencias significativas, excepto en la longitud de las plantas (cm) lo cual al parecer resulta de las diferencias genéticas entre las variedades (Tabla 4).

Oliveira et al. (2000) reportaron la aclimatación exitosa de plantas de yuca obtenidas por cultivo de tejidos; otros autores, en cambio, informaron una elevada mortalidad en esta etapa (Zimmerman et al., 2007; Marín et al., 2008), que puede llegar hasta el $95 \%$ si no se realiza con la tecnología adecuada (Segovia et al., 2002; Albarrán et al., 2011). En esta investigación, los altos porcentajes de supervivencia alcanzados pueden deberse a que se hizo una pre-aclimatación, según lo recomendado por Bonilla et al. (2015) antes de exponer a las plantas a las condiciones naturales.

La presente investigación permitió determinar las condiciones esenciales para la micropropagación de las variedades INIAP Portoviejo 650 e INIAP Portoviejo 651 (Fig. 1).

Los resultados obtenidos de la propagación de estos genotipos, de interés para la producción en Ecuador, pueden ser útiles en su multiplicación acelerada con vistas a proporcionar propágulos a los agricultores. No obstante, será necesario realizar investigaciones más amplias para estandarizar estos procedimientos a una escala masiva, considerando las particularidades de cada una de las variedades estudiadas.

\section{CONCLUSIONES}

La adición de amoxicilina al medio de cultivo $\left(0,5 \mathrm{mg} \mathrm{L}^{-1}\right)$ evitó la contaminación de los explantes. Los mejores resultados en las variables analizadas en la multiplicación in vitro se consiguieron adicionando al medio $0,5 \mathrm{mg}$ $\mathrm{L}^{-1} \mathrm{BAP}+0,30 \mathrm{mg} \mathrm{L}^{-1} \mathrm{GA}_{3}$. La adición al medio MS de $1 \mathrm{mg} \mathrm{L}^{-1} \mathrm{ANA}+0,01 \mathrm{mg} \mathrm{L}^{-1} \mathrm{GA}_{3}$, favoreció el enraizamiento in vitro de los explantes. Las vitroplantas mostraron buen comportamiento en la aclimatación ex vitro.

Tabla 4. Longitud de la planta, diámetro del tallo, número de brotes y número de hojas en la aclimatación de vitroplantas de yuca.

Table 4. Plant height, stem diameter, and number of shoots and leaves in the acclimatization of cassava glass plants.

\begin{tabular}{lcccc}
\hline $\begin{array}{l}\text { Fuente de } \\
\text { variación }\end{array}$ & $\begin{array}{c}\text { Longitud de la } \\
\text { planta }(\mathbf{c m})\end{array}$ & $\begin{array}{c}\text { Diámetro del } \\
\text { tallo }(\mathbf{c m})\end{array}$ & $\begin{array}{c}\text { Número de } \\
\text { brotes }\end{array}$ & $\begin{array}{c}\text { Número } \\
\text { de hojas }\end{array}$ \\
\hline INIAP 650 & 2,15 & 0,85 & 3,90 & 4,10 \\
INIAP 651 & 2,75 & 1,08 & 4,60 & 4,20 \\
p-valor & $0,0065^{*}$ & 0,064 NS & 0,26 NS & 0,57 NS \\
CV $(\%)$ & 17,79 & 19 & 28,74 & 22,63 \\
\hline
\end{tabular}




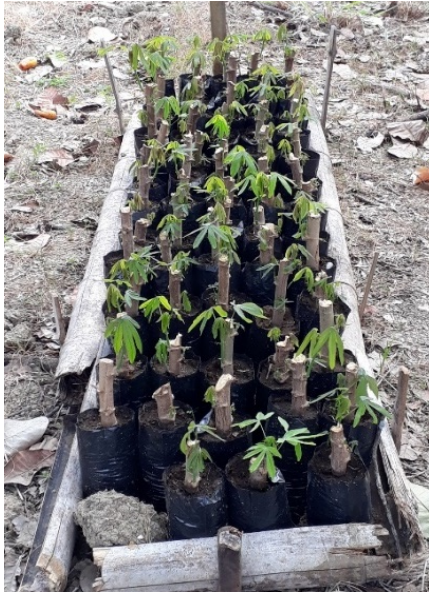

A

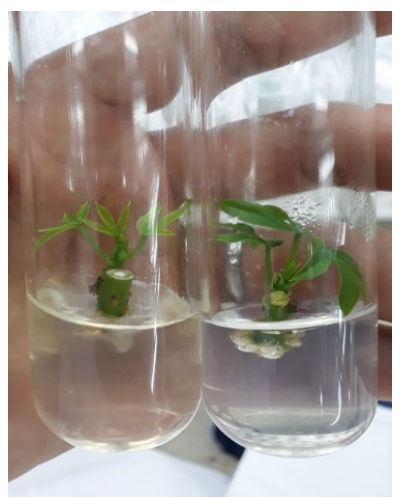

B

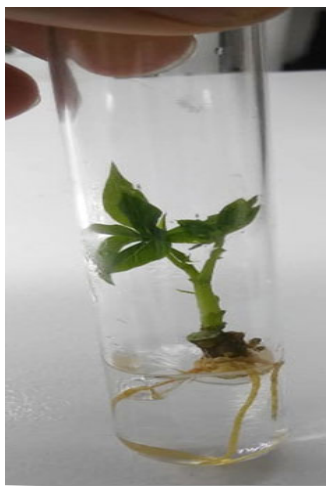

D

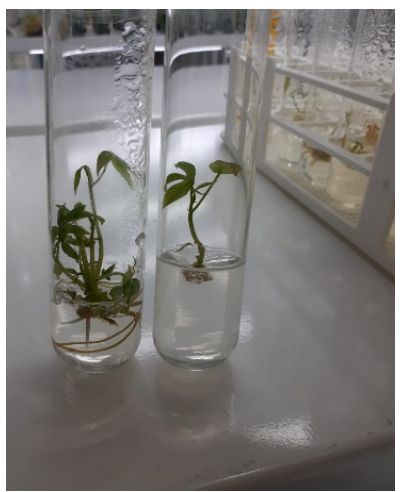

C

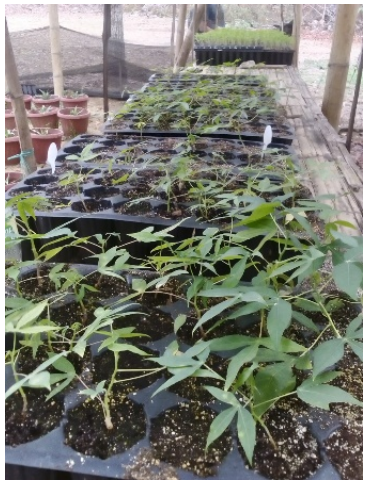

$\mathbf{E}$

Fig. 1. Procedimiento de micropropagación en yuca para las variedades INIAP Portoviejo 650 e INIAP Portoviejo 651. A: banco de donantes; B: establecimiento; C: multiplicación: D: enraizamiento; E: aclimatación.

Fig. 1. Micropropagation procedure in cassava for the varieties INIAP Portoviejo 650 and INIAP Portoviejo 651. A: donor bank; B: establishment; C: multiplication; D: rooting; E: acclimatization.

\section{LITERATURA CITADA}

Abreu, E., M. del Castillo, G. del Sol, y G. González. 2016. Efecto de antibióticos en la propagación in vitro de Agave fourcroydes Lem. Biotecnología Vegetal 16(1):31-36.

Akinbo, O., M. Labuschagne, and M. Fregene. 2010. Embryo rescue as a method to develop and multiply a backcross population of cassava (Manihot esculenta Crantz) from an interspecific cross of Manihot esculenta ssp. flabellifolia. Afr. J. Biotechnol. 9(42):70587062.

Albarrán, J., F. Fuenmayo., M. Fuchs, G. Martínez, A. Rodríguez, E. Manzanilla, et al. 2011. Biotechnological strategies for germplasm conservation in the INIA-CENIAP, Venezuela. Case: cassava and Musa. Agronomía Tropical (Maracay) 61(1):85-94.
Ali, S., N. Khan, F. Nouroz, S. Erum, W. Nasim, and M.A. Shahid. 2018. In vitro effects of $\mathrm{GA}_{3}$ on morphogenesis of CIP potato explants and acclimatization of plantlets in field. In Vitro Cell Dev. Biol.-Plant. 54(1):104-111.

Basail, M. 2015. Influencia de la concentración de reguladores del crecimiento para la multiplicación del clon de malanga "INIVITMX-2007". Agricultura Tropical 1(1):33-38.

Beltrano, J., y D. Gimenez. 2015. Cultivo en hidroponía. 1a. ed.. Editorial UNLP, La Plata, Argentina.

Berhanu, R., and T. Feyissa. 2013. Factors affecting in vitro propagation of cassava (Manihot esculenta Crantz.) Euphorbiaceae, varieties of 'Kello'and 'Qulle'. Ethiop. J. Biol. Sci. 12(1):25-39. 
Bonilla, M., J. Pachón, y M. Moreno. 2015. Propagación in vitro de yuca (Manihot esculenta Crantz) variedad brasilera: una alternativa de producción de semilla libre de patógenos para el Meta, Colombia. Cuadernos de Recursos Fitogenéticos Neotropicales 5(6):48-60.

Collado, L., C. Bermúdez, L. García, N. Veitía, A. Martirena, D. Torres, et al. 2004. Propagación in vitro de tres cultivares de yuca. Instituto de Biotecnología de las Plantas, Santa Clara, Cuba.

Elmongy, M., Y. Cao, H. Zhou, and Y. Xia. 2018. Root development enhanced by using indole-3-butyric acid and naphthalene acetic acid and associated biochemical changes of in vitro azalea microshoots. Journal of Plant Growth Regulation 37(3):813-825.

FAO. 2014. Regional Conference on Cassava in the Caribbean and Latin America. Conference Report 10-12 February 2014. Food and Agriculture Organization of the United Nations (FAO), Rome, Italy.

FAOSTAT. 2018. Food and Agriculture Organization. Corporate Statistical Database. Available at http://www.fao.org/faostat/ en/\#home [Accessed 23 March 2020].

Héctor, E., A. Torres, S. Algoe, M. Cabañas, and A. López. 2007. Propagación in vitro del plátano macho (Musa spp. AAB) clon Sobrino con los bioestimulantes cubanos BB-6 y Biostan como sustitutos de los reguladores del crecimiento. Cultivos Tropicales 28(1):13-18.

INEC. 2018. Estadística de la producción y áreas de siembra de la yuca en Manabí. Informe Técnico de Estadísticas de Siembra y Producción de yuca en Manabí. Instituto Nacional de Estadísticas y Censos (INEC), Quito, Ecuador.

INIAP. 2008. Innovaciones para emprendimiento de yuca (Manihot esculenta Crantz) y camote (Ipomoea batatas $\mathrm{L}$ ) en la seguridad y soberanía alimentaria, y oportunidades de mercado para pequeños/as productores/as emprendedores de Manabí-Ecuador. Instituto Nacional Autónomo de Investigaciones Agropecuarias (INIAP), Quito, Ecuador.

Kidulile, C.E., A.E. Alakonya, J.C. Ndunguru, and E.M. Ateka. 2018. Cost effective medium for in vitro propagation of Tanzanian cassava landraces. Afr. J. Biotechnol. 17(25):787-794.

Laynez, J.A., and M.C. Sánchez. 2006. Desinfección de ápices de yuca (Manihot esculenta Crantz) cv. 'Querepa rosada' con hipoclorito de sodio. Revista UDO Agrícola 6(1):60-66.
Leelavathy, S., and P.D. Sankar. 2016. Curbing the menace of contamination in plant tissue culture. J. Pure and Appl. Microbiol. 10(3):2145-2152.

Liu, J., Q. Zheng, Q. Ma, K.K. Gadidasu, and P. Zhang. 2011. Cassava genetic transformation and its application in breeding. J. Integr. Plant Biol. 53(7):552-569.

Lolaei,A., N. Teymouri, R, Bemana, A. Kazempour, and S. Aminian. 2013. Effect of gibberellin on vegetative and sexual growth and fruit quality of strawberry (Fragaria×Ananassa Duch. cv. Selva and Qeen elisa). Int. J. Agric. Crop Sci. 5(14):1508-1513.

Mafla, G., J. Roa, E. Aranzales, y D. Debouck. 2010. Manual de procedimientos para la conservación in vitro del germoplasma del género Manihot. Centro Internacional de Agricultura Tropical, Cali, Colombia.

Marín, A., D. Perdomo, J.G. Albarrán, F. Fuenmayor, y C. Zambrano. 2008. Evaluación agronómica, morfológica y bioquímica de clones élites de yuca a partir de vitroplantas. Interciencia 33(5):365-371.

Marín, A., J.G. Albarrán, F. Fuenmayor, and D. Perdomo. 2009. Evaluación del efecto de los reguladores de crecimiento en la regeneración in vitro de cinco cultivares élites de yuca (Manihot esculenta Crantz). Revista Científica UDO Agrícola 9(3):556-562.

Mehmood, A., A.H. Shah, A.H. Shah, M. Sajid, and $\mathrm{H}$. Ahmad. 2016. Investigation of $\mathrm{GA}_{3}$ effect on in vitro micro propagation of potato varieties. Int. J. Agri. Res. 9(2):190-197.

Mongomake, K., O. Doungous, B. Khatabi, and V.N. Fondong. 2015. Somatic embryogenesis and plant regeneration of cassava (Manihot esculenta Crantz) landraces from Cameroon. SpringerPlus 4:477. https://doi.org/10.1186/ s40064-015-1272-4

Moura, I. R., and M. Ferreira. 2004. Influence of different growth regulators on cassava (Manihot esculenta Crantz) in vitro propagation from node cultures. Revista de Ciencias Agrarias (Portugal). Available at http://agris.fao.org/agris-search/search. do? recordID=PT2005000176 (Accessed 25 March 2020)

Murashige, T., and F. Skoog. 1962. A revised medium for rapid growth and bioassays with tobacco tissue culture. Physiologia Plantarum 15(3):473-497.

Ogero, K., G.N. Mburugu, M. Mwangi, O. Ombori, and M. Ngugi. 2012. In vitro micropropagation of cassava through low cost tissue culture. Asian J. Agr. Sci. 4(3):205209. 
Oliveira, R.P., T. Gomes, and A. Vilarinhos. 2000. Avaliação de um sistema de micropropagação massal de variedades de mandioca. Pesq. Agrop. Bras. 35(12):2329-2334.

Ortega, L.D., J. Ocampo, C. Martínez, A. Pérez, y J. Sánchez. 2013. Efecto de las giberelinas sobre el crecimiento y calidad de plántulas de tomate. Biotecnia 15(3):56-60.

Palla, K., R. Beasley, and P. Pijut. 2013. In vitro culture and rooting of Diospyros virginiana $\mathrm{L}$. HortScience 48(6):747-749.

Pereira, N., B. Ferreira, E. de Carvalho, y C. Damiani. 2018. Application of Chlorella sorokiniana (Chlorophyceae) as supplement and/or an alternative medium for the in vitro cultivation of Schomburgkia crispa (Orchidaceae). Journal of Applied Phycology 30(4):2347-2358.

Rabbani, A., B. Askari, N. Abbasi, M. Bhatti, and A. Quraishi. 2001. Effect of growth regulators on in vitro multiplication of potato. Int. J. Agric. Biol. 3(2):181-182.

Reddy, L.J., B. Jose, J. Anjana, and T. Ruveena. 2010. Evaluation of antibacterial activity of Trichosanthes cucumerina L. and Cassia didymobotrya Fres. leaves. Int. J. Pharm. Sci. 2(4):153-155.

Roca, W. 1984. Cassava. p. 403-420. In Sharp W. R., D. Evans, P. Ammirato and Y. Yamada (eds.) Handbook of plant cell culture. Crop species. Macmillan, New York, USA.

Segovia, R.J., A. Bedoya, W. Triviño, H. Ceballos, G. Gálvez, y B. Ospina. 2002. Metodología para el endurecimiento masivo de 'vitroplantas' de yuca. En: La yuca en el tercer milenio: sistemas modernos de producción, procesamiento, utilización y comercialización. Ospina, B. y Ceballos, H. (eds.) Centro Internacional de Agricultura Tropical, Cali, Colombia.

Souza, G., M. Campos., e P. Clemente. 2007. Contaminação microbiana na propagação in vitro de Cattleya walkeriana e Schomburgkia crispa. Rev. Brasil. Biociênc. 5:405-407.

Suárez, L, and M.M. Hernández. 2015. Efecto del Pectimorf $^{\circledast}$ en el cultivo de ápices de plantas in vitro de yuca (Manihot esculenta Crantz), clones "CMC-40" y "Señorita". Cultivos Tropicales 36(4):55-62.
Taiz, L, and E. Zeiger, 2006. Fisiología vegetal (Vol. 10). Universitat Jaume I. Castellón de la Plana, España.

Taylor, N., E. Gaitán, T. Moll, B. Trauterman, T. Jones, A. Pranjal, et al. 2012. A highthroughput platform for the production and analysis of transgenic cassava (Manihot esculenta) plants. Trop. Plant Biol. 5(1):127139.

Techeira, N., L. Sívoli, B. Perdomo, A. Ramírez, y F. Sosa. 2014. Caracterización fisicoquímica, funcional y nutricional de harinas crudas obtenidas a partir de diferentes variedades de yuca (Manihot esculenta Crantz), batata (Ipomoea batatas Lam) y ñame (Dioscorea alata), cultivadas en Venezuela. Interciencia 39(3):191-197.

Ulloa, A.J. 2017. Efecto de 6-bencil aminopurina y ácido naftalenacético en la producción in vitro de segmentos nodales de yuca (Manihot esculenta Crantz). Tesis de Ingeniero Agrónomo, Universidad Zamorano, Honduras.

Xu, Q., S. Krishnan, E. Merewitz, J. Xu, and B. Huang. 2016. Gibberellin-regulation and genetic variations in leaf elongation for tall fescue in association with differential gene expression controlling cell expansion. Scientific Reports 6(30258). https://doi. org/10.1038/srep30258

Yandia, S.P., C. Gandonou, S. Sembala, I. Zinga, and T. Fatiou. 2018. Response of four cultivars of cassava (Manihot esculenta Crantz) plantlets free of cassava mosaic virus to micropropagation in different media. Afr. J. Biotechnol. 17(1):9-16.

Zimmerman, T.W., K. Williams, L, Joseph, J. Wiltshire, and J. Kowalski. 2007. Rooting and acclimatization of cassava (Manihot esculenta) ex vitro. Acta Hort. 733:735-740. 